\title{
ON THE ALGEBRAIC INDEPENDENCE OF THE VALUES OF SOME $E$-FUNCTIONS
}

\author{
KEIJO VÄÄNÄNEN
}

In the following $\mathbf{Q}$ denotes the field of rational numbers, $\mathbf{C}$ the field of complex numbers and $\mathbf{C}(z)$ the field of rational functions of $z$ with coefficients in $\mathbf{C}$.

1. In this paper we shall consider the functions

(1) $u_{0, i}(z, \nu, \mu)=(1 / i !) \frac{\partial^{i}}{\partial \mu^{i}} K_{\nu, \mu}(z), \quad u_{i, 0}(z, \nu, \mu)=(1 / i !) \frac{\partial^{i}}{\partial \nu^{i}} K_{\nu, \mu}(z)$, where $K_{v, \mu}$ is the Kummer function

$$
\begin{gathered}
K_{\nu, \mu}(z)=\sum_{n=0}^{\infty}(\mu(\mu+1) \ldots(\mu+n-1) / n ! \nu(\nu+1)(\nu+n-1)) z^{n}, \\
\nu, \mu \neq 0,-1,-2, \ldots
\end{gathered}
$$

The function values $K_{v, \mu}(\alpha), K_{v, \mu}^{\prime}(\alpha)$, where $\alpha \neq 0$ is an algebraic number and $\nu, \mu \in \mathbf{Q}$, have been considered in many papers (see [1], [3]). We shall now prove the following theorems. (Throughout this paper we denote differentiation with respect to $z$ by a dash.)

Theorem 1. Let $\alpha \neq 0$ be an algebraic number, and let $\nu$ and $\mu$ be rational numbers satisfying the conditions

$$
\nu \neq 0,-1,-2, \ldots ; \quad \mu, \mu-\nu \neq 0, \pm 1, \pm 2, \ldots
$$

Then the numbers of each of the two sets

$$
\begin{array}{llll}
u_{0, k}(\alpha, \nu, \mu), & u_{0, k}^{\prime}(\alpha, \nu, \mu), & e^{\alpha}, & k=0,1, \ldots, n ; \\
u_{i, 0}(\alpha, \nu, \mu), & u_{i, 0}^{\prime}(\alpha, \nu, \mu), & e^{\alpha}, & i=0,1, \ldots, m,
\end{array}
$$

are algebraically independent.

Theorem 2. Let the conditions of Theorem 1 be satisfied. Further, let $\nu \neq 1,2, \ldots$. Then the $2(m+n)+3$ numbers 


$$
\begin{aligned}
u_{i, 0}(\alpha, \nu, \mu), & u_{i, 0}^{\prime}(\alpha, \nu, \mu), u_{0, k}(\alpha, \nu, \mu), u_{0, k}^{\prime}(\alpha, \nu, \mu), e^{\alpha}, \\
& i=0,1, \ldots, m, \quad k=1,2, \ldots, n,
\end{aligned}
$$

are algebraically independent.

2. In the proofs of our theorems we shall use the method of Siegel and Sidlovskil. Thus we shall prove the following lemmas.

Lemma 1. Let $v$ and $\mu$ satisfy the conditions of Theorem 1. Further, let $\gamma \neq 0$ be a rational number. Then the $2 n+3$ functions

$$
u_{0, k}(z, \nu, \mu), \quad u_{0, k}^{\prime}(z, \nu, \mu), \quad e^{\gamma z}, \quad k=0,1, \ldots, n,
$$

are algebraically independent over $\mathbf{C}(z)$.

Lemma 2. Let the conditions of Lemma 1 be satisfied. Then the $2 m+3$ functions

$$
u_{i, 0}(z, \nu, \mu), \quad u_{i, 0}^{\prime}(z, \nu, \mu), \quad e^{\gamma z}, \quad i=0,1, \ldots, m,
$$

are algebraically independent over $\mathbf{C}(z)$.

Lemma 3. Let $\nu$ and $\mu$ satisfy the conditions of Theorem 2. Further, let $\gamma \neq 0$ be a rational number. Then the $2(m+n)+3$ functions

$$
\begin{aligned}
u_{i, 0}(z, \nu, \mu), & u_{i, 0}^{\prime}(z, \nu, \mu), u_{0, k}(z, \nu, \mu), u_{0, k}^{\prime}(z, \nu, \mu), e^{\gamma z}, \\
& i=0,1, \ldots, m, \quad k=1,2, \ldots, n,
\end{aligned}
$$

are algebraically independent over $\mathbf{C}(z)$.

3. We begin by proving Lemma 1. For the sake of simplicity we put $u_{0, k}(z, \nu, \mu)=u_{k}, \quad k=0,1, \ldots$, and use in the following the notations $u(k)$ and $u^{\prime}(k)$ to denote respectively the functions $u_{0}, u_{1}, \ldots, u_{k}$ and $u_{0}^{\prime}, u_{1}^{\prime}, \ldots, u_{k}^{\prime}$.

The function $u_{0}=K_{v, \mu}(z)$ satisfies the differential equation

$$
u_{0}^{\prime \prime}+(\nu / z-1) u_{0}^{\prime}-(\mu / z) u_{0}=0 .
$$

Thus we can deduce that the functions $u(n)$ satisfy the following system of differential equations,

(5) $u_{k}^{\prime \prime}+(\nu / z-1) u_{k}^{\prime}-(\mu / z) u_{k}-(1 / z) u_{k-1}=0, \quad u_{-1} \equiv 0, \quad k=0,1, \ldots, n$.

It follows that if $P=P\left(z, u(n), u^{\prime}(n), e^{\gamma z}\right)$ is a polynomial in $z$, $u(n), u^{\prime}(n), e^{\gamma z}$, then $z P^{\prime}$ will also be a polynomial in the same variables.

In the proof of Lemma 1, which will be performed by induction, we need the following lemma.

Lemma 4. Let us assume that we have an irreducible polynomial $P=P\left(z, u(n), u^{\prime}(n), e^{\gamma z}\right)$ satisfying 


$$
P=\sum_{i=0}^{m} P_{i} e^{i \gamma z}=0,
$$

where $P_{i}, i=0,1, \ldots, m$, are polynomials in $z, u(n), u^{\prime}(n)$ such that $P_{m} \equiv 0$. If the functions $u(n), u^{\prime}(n)$ are algebraically independent over $\mathbf{C}(z)$, then $P$ must satisfy, identically in $z, u(n), u^{\prime}(n), e^{\gamma z}$, the equation

$$
z P^{\prime}=(a z+b) P, \quad a, b \in \mathbf{Q},
$$

and, further, $P$ must be of the form

$$
P=P_{m} e^{m \gamma z}+P_{0}, \quad m \geqq 1,
$$

where the polynomials $P_{m}$ and $P_{0}$ are homogeneous with respect to $u(n)$, $u^{\prime}(n)$ and one of them is a polynomial in z alone.

For the proof of this lemma we refer to [7] (pp. 5-6).

First let $n=0$. The algebraic independence of the functions $u_{0}, u_{0}^{\prime}$ is proved in [3]. If the functions $u_{0}, u_{0}^{\prime}, e^{\gamma z}$ were algebraically dependent over $\mathbf{C}(z)$, then we should have an equation of the form (6). By Lemma 4, this yields

$$
P=P_{m} e^{m \gamma z}+P_{0}=0,
$$

where $P$ satisfies (7), $P_{m}$ and $P_{0}$ are homogeneous polynomials with respect to $u_{0}, u_{0}^{\prime}$ such that $P_{m}=c_{m} z^{b}$ or $P_{0}=c_{0} z^{b}$ with non-zero constants $c_{m}, c_{0}$. Further, we can assume that $\gamma>0$.

$1^{\circ}$. Let $P_{m}=c_{m} z^{b}$. Then $a=m \gamma$, so that $P_{0}$ satisfies, by (7), the differential equation

$$
z P_{0}^{\prime}=(m \gamma z+b) P_{0}
$$

If we denote

$$
P_{0}=\sum_{i=0}^{l} A_{i} u_{0}^{l-i} u_{0}^{\prime i}
$$

where $A_{i}$ are polynomials in $z$, then (9) and the algebraic independence of the functions $u_{0}, u_{0}^{\prime}$ implies that

$$
\begin{gathered}
\text { (10) } z A_{i}^{\prime}+(l-i+1) z A_{i-1}+(i+1) \mu A_{i+1}=((m \gamma-i) z+b+i \nu) A_{i}, \\
i=0,1, \ldots, l, \quad A_{-1} \equiv A_{l+1} \equiv 0 .
\end{gathered}
$$

Let $k_{i}$ be the degree of the polynomial $A_{i}, i=0,1, \ldots, l$. If $m \gamma-i \neq 0$ $\forall i=0,1, \ldots, l$, then $(10)$ with $i=0,1, \ldots, l-1$ gives

$$
k_{i}=k_{0}+i, \quad i=0,1, \ldots, l .
$$

Thus $k_{l-1}+1=k_{l}$. But if $i=l$, then (10) gives $k_{l-1}=k_{l}$. This contradiction means that there exists one $i$, say $i_{0}$, such that $m \gamma=i_{0}$. Thus (8) must be of the form 


$$
c_{m} z^{b} e^{i_{0} z}+\sum_{i=0}^{l} A_{i} u_{0}^{l-i} u_{0}^{\prime i}=0
$$

We now denote

$$
B_{v, \mu}(z)=e^{-z / 2} u_{0}, \quad U_{v, \mu}(z)=z^{(v-1) / 2} B_{v, \mu}(z) .
$$

From the proof of Lemma 7 of [1] it follows that the functions $U_{v, \mu}(z)$, $U_{v, \mu}^{\prime}(z), \quad e^{z / 2}$ are algebraically independent over $\mathbf{C}(z)$. Since $v \in \mathbf{Q}$, this implies the algebraic independence of the functions $B_{v, \mu}(z), B_{v, \mu}^{\prime}(z), e^{z / 2}$. From (11) it follows that

$$
c_{m} z^{b} e^{i_{0} z}+e^{l z / 2} \sum_{i=0}^{l} A_{i} B_{v, \mu}^{l-i}\left(B_{v, \mu} / 2+B_{v, \mu}^{\prime}\right)^{i}=0 .
$$

Since $B_{v, \mu}(z), \quad B_{v, \mu}^{\prime}(z), \quad e^{z / 2}$ are algebraically independent over $\mathbf{C}(z)$, it follows that $2 i_{0}=l$. Further, we must have $l \geqq 1$. This leads to a contradiction.

$2^{\circ}$. If $P_{0}=c_{0} z^{b}$, then $a=0$, and $P_{m}$ satisfies the differential equation

$$
z P_{m}^{\prime}=(-m \gamma z+b) P_{m} .
$$

If we repeat the reasonings used in the early steps of the first case, we get

$$
-m \gamma=i_{0}, \quad i_{0} \in\{0,1, \ldots, l\} .
$$

This is impossible, since $-m \gamma<0$. Thus Lemma 1 is true when $n=0$.

Now let us assume that this lemma holds when $n=k$. Next we shall prove that this implies the truth of Lemma 1 with $n=k+1$. We split the proof into three steps, called in the following $\mathrm{A}, \mathrm{B}$ and $\mathrm{C}$.

4. Step A. We define

$$
t=u_{0} u_{k+1}^{\prime}-u_{0}^{\prime} u_{k+1}
$$

and prove that the functions $u(k), u^{\prime}(k), t$ are algebraically independent over $\mathbf{C}(z)$. If we assume, against this, that these functions are algebraically dependent, then we have

$$
P=\sum_{i=0}^{m} P_{i} t^{i}=0
$$

where $P$ is an irreducible polynomial in $z, u(k), u^{\prime}(k), t$, and $P_{i}$, $i=0,1, \ldots, m$, are polynomials in $z, u(k), u^{\prime}(k)$ such that $P_{m} \neq 0$. By induction hypothesis, $m \geqq 1$.

By (5), we obtain

$$
z t^{\prime}=(z-v) t+u_{0} u_{k} .
$$


From this and (5) it follows that $z P^{\prime}$ is also a polynomial in $z, u(k)$, $u^{\prime}(k), t$. Further, by (12), $z P^{\prime}=0$. Thus the induction hypothesis implies that the polynomial $z P^{\prime}$ must be divisible by the polynomial $P$. Since the degree of $z P^{\prime}$ with respect to $t$ does not exceed that of $P$, there is a polynomial $S$ in $z, u(k), u^{\prime}(k)$ such that

$$
z P^{\prime}=S P
$$

identically in $z, u(k), u^{\prime}(k), t$. If we compare the coefficients of $t^{m}$ in (13), we obtain an identity

$$
z P_{m}^{\prime}+m(z-v) P_{m}=S P_{m} \text {. }
$$

Since the degree of $z P_{m}^{\prime}$ with respect to $u(k), u^{\prime}(k)$ does not exceed that of $P_{m}$, and the degree of $z P_{m}^{\prime}$ with respect to $z$ exceeds that of $P_{m}$ at most by one, it follows that $S=a z+b$ with constants $a$ and $b$. Thus the identity (13) takes the form

$$
z P^{\prime}=(a z+b) P .
$$

Here we can assume, by Lemma 10 of [4], that $a, b \in \mathbf{Q}$.

If $\bar{u}_{0}, \bar{u}_{1}, \ldots, \bar{u}_{k+1}$ is another solution of (5) with $n=k+1$, and $\bar{t}=\bar{u}_{0} \bar{u}_{k+1}^{\prime}-\bar{u}_{0}^{\prime} \bar{u}_{k+1}$, then

$$
z \bar{t}^{\prime}=(z-v) \bar{t}+\bar{u}_{0} \bar{u}_{k} .
$$

Thus (14) holds even when we replace the solution $u(k+1)$ by any other solution $\bar{u}(k+1)$. Now denote $\bar{u}(k)=u(k), \bar{u}_{k+1}=u_{k+1}+\sigma y$, where $y$ is a solution of (4), linearly independent of $u_{0}$, and $\sigma$ is an arbitrary constant. By integrating (14) we then obtain

$$
\sum_{i=0}^{m} P_{i}\left(t+\sigma\left(u_{0} y^{\prime}-u_{0}^{\prime} y\right)\right)^{i}=c(\sigma) z^{b} e^{a z} .
$$

When we differentiate this equation with respect to $\sigma$ and put $\sigma=0$, we obtain

$$
\left(u_{0} y^{\prime}-u_{0}^{\prime} y\right) \sum_{i=1}^{m} i P_{i} t^{i-1}=c^{\prime}(0) z^{b} e^{a z}
$$

Now $u_{0} y^{\prime}-u_{0}^{\prime} y=c z^{-v} e^{z}, \quad c \neq 0$, and this gives the following equation

$$
\sum_{i=1}^{m} i P_{i} t^{i-1}=c^{\prime}(0) c^{-1} z^{b+v} e^{(a-1) z} .
$$

First let $m>1$. If $c^{\prime}(0)=0$, we have $\sum_{i=1}^{m} i P_{i} t^{i-1}=0$. This is impossible, since $P$ is irreducible. If $c^{\prime}(0) \neq 0$, then $b+\nu$ must be a non-negative integer. If $a=1$, then we have the same contradiction as before. If $a \neq 1$, then we can eliminate $t$ from the equations (12) and 
(15), and thus obtain an algebraic relation over $\mathbf{C}(z)$ between the functions $u(k), u^{\prime}(k), e^{(a-1) z}$. This conflicts with the induction hypothesis. Thus $m=1$, and (15) has the form

$$
P_{1}=c^{\prime}(0) c^{-1} z^{b+v_{e}}(a-1) z .
$$

Thus $b+v$ must again be a non-negative integer. Further, it follows from the induction hypothesis that $a=1$. This implies that

$$
P_{1}=c_{0}^{z b+\nu}, \quad c_{0} \neq 0 .
$$

We have now proved that the equation (12) has the form

$$
P=c_{0} z^{b+v} t+P_{0}=0 \text {. }
$$

Now we shall analyze the polynomial $P_{0}$. Denote

$$
P_{0}=\sum_{i=0}^{l} H_{i}
$$

where each $H_{i}$ is a homogeneous polynomial of degree $i$ with respect to $u(k), u^{\prime}(k)$. Replace now in (14) $u_{i}$ by $\bar{u}_{i} \dot{=} \sigma u_{i}, i=0,1, \ldots, k+1$, with an arbitrary constant $\sigma$. By integrating (14) we then obtain the equation

$$
c_{0} z^{b+v} \sigma^{2} t+\sum_{i=0}^{l} \sigma^{i} H_{i}=c(\sigma) z^{b} e^{z} .
$$

When we differentiate this equation twice with respect to $\sigma$, and put $\sigma=0$, we have

$$
2 c_{0} z^{b+v} t+2 H_{2}=c^{\prime \prime}(0) z^{b} e^{z} .
$$

Thus $H_{2}-P_{0}=c^{\prime \prime}(0) z^{b} e^{z} / 2$, and this together with the induction hypothesis gives the result $P_{0} \equiv H_{2}$. Thus $P_{0}$ is a homogeneous polynomial of degree two with respect to $u(k), u^{\prime}(k)$.

Let $s=[k / 2]$. If $s>0$, put $\bar{u}(k-1)=u(k-1), \quad \bar{u}_{k}=$ $u_{k}+\sigma u_{0}, \bar{u}_{k+1}=u_{k+1}+\sigma u_{1}$ with an arbitrary constant $\sigma$. By putting

$$
P_{0}=A_{1}+P_{10} u_{k}+P_{01} u_{k}^{\prime}+P_{20} u_{k}^{2}+P_{11} u_{k} u_{k}^{\prime}+P_{02} u_{k}^{\prime 2},
$$

where $A_{1}$ and $P_{i j}$ do not contain $u_{k}$ or $u_{k}^{\prime}$, and integrating (14), we obtain

$$
\begin{gathered}
c_{0} z^{b+v}\left(t+\sigma\left(u_{0} u_{1}^{\prime}-u_{0}^{\prime} u_{1}\right)\right)+A_{1}+P_{10}\left(u_{k}+\sigma u_{0}\right)+P_{01}\left(u_{k}^{\prime}+\sigma u_{0}^{\prime}\right)+ \\
+P_{20}\left(u_{k}+\sigma u_{0}\right)^{2}+P_{11}\left(u_{k}+\sigma u_{0}\right)\left(u_{k}^{\prime}+\sigma u_{0}^{\prime}\right)+P_{02}\left(u_{k}^{\prime}+\sigma u_{0}^{\prime}\right)^{2}=c(\sigma) z^{b} e^{z} .
\end{gathered}
$$

When we differentiate this equation with respect to $\sigma$, put $\sigma=0$ and again invoke the induction hypothesis, we obtain the result 


$$
\begin{aligned}
P & =c_{0} z^{b+v}\left(t+\left(u_{1} u_{k}^{\prime}-u_{1}^{\prime} u_{k}\right)\right)+a_{1}(z)\left(u_{0} u_{k}^{\prime}-u_{0}^{\prime} u_{k}\right)+A_{1} \\
& =\sum_{l=0}^{1} a_{l} \sum_{j=0}^{1-l}\left(u_{j} u_{k+1-l-j}^{\prime}-u_{j}^{\prime} u_{k+1-l-j}\right)+A_{1},
\end{aligned}
$$

where $a_{0}=c_{0} z^{b+v}, a_{1}=a_{1}(z)$ is a polynomial in $z$, and $A_{1}$ is a homogeneous polynomial of degree two with respect to $u(k-1), u^{\prime}(k-1)$.

Next, let us assume that for some $i<s$ we have

$$
P=\sum_{l=0}^{i} a_{l} \sum_{j=0}^{i-l}\left(u_{j} u_{k+1-l-j}^{\prime}-u_{j}^{\prime} u_{k+1-l-j}\right)+A_{i},
$$

where $A_{i}$ is a homogeneous polynomial of degree two with respect to $u(k-i), u^{\prime}(k-i)$, and $a_{l}=a_{l}(z)$ are polynomials in $z$. Let us again denote

$$
A_{i}=A_{i+1}+P_{10} u_{k-i}+P_{01} u_{k-i}^{\prime}+P_{20} u_{k-i}^{2}+P_{11} u_{k-i} u_{k-i}^{\prime}+P_{02} u_{k-i}^{\prime 2} .
$$

The substitution $\quad \bar{u}(k-i-1)=u(k-i-1), \quad \bar{u}_{k-i}=u_{k-i}+\sigma u_{0}$, $\bar{u}_{k+1-i}=u_{k+1-i}+\sigma u_{1}, \ldots, \bar{u}_{k+1}=u_{k+1}+\sigma u_{i+1}$ in (14) now gives

$$
\begin{aligned}
& \sum_{l=0}^{i} a_{i} \sum_{j=0}^{i-l}\left(u_{j} u_{k+1-l-j}^{\prime}-u_{j}^{\prime} u_{k+1-l-j}+\sigma\left(u_{j} u_{i+1-l-j}^{\prime}-u_{j}^{\prime} u_{i+1-l-j}\right)\right) \\
&(17) \quad+A_{i+1}+P_{10}\left(u_{k-i}+\sigma u_{0}\right)+P_{01}\left(u_{k-i}^{\prime}+\sigma u_{0}^{\prime}\right)+P_{20}\left(u_{k-i}+\sigma u_{0}\right)^{2}+ \\
&+P_{11}\left(u_{k-i}+\sigma u_{0}\right)\left(u_{k-i}^{\prime}+\sigma u_{0}^{\prime}\right)+P_{02}\left(u_{k-i}^{\prime}+\sigma u_{0}^{\prime}\right)^{2}=c(\sigma) z^{b} e^{z} .
\end{aligned}
$$

By differentiating this equation with respect to $\sigma$ and putting $\sigma=0$, we obtain

$$
P=\sum_{l=0}^{i+1} a_{l} \sum_{j=0}^{i+1-l}\left(u_{j} u_{k+1-l-j}^{\prime}-u_{j}^{\prime} u_{k+1-l-j}\right)+A_{i+\mathbf{1}} .
$$

It follows that

$$
P=\sum_{l=0}^{s} a_{l} \sum_{j=0}^{s-l}\left(u_{j} u_{k+1-l-j}^{\prime}-u_{j}^{\prime} u_{k+1-l-j}\right)+A_{s},
$$

where again $A_{s}$ is a homogeneous polynomial of degree two with respect to $u(k-s), u^{\prime}(k-s)$. We now have two possibilities, either $2 s=k$ or $2 s+1=k$.

$1^{\circ} .2 s=k$. Denote

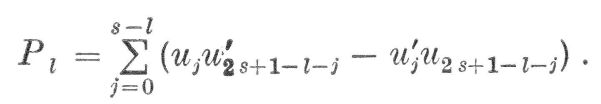

Since the polynomial $P=\sum_{l=0}^{s} a_{l} P_{l}+A_{s}$ satisfies the differential equation (14) with $a=1$, we obtain 


$$
\sum_{l=0}^{s}\left(z a_{l}^{\prime}-(b+v) a_{l}\right) P_{l}+\sum_{l=0}^{s} a_{l} u_{s-l} u_{s}+z A_{s}^{\prime}=(z+b) A_{s} .
$$

We have $a_{0}=c_{0} z^{b+v}$, and so $z a_{0}^{\prime}-(b+v) a_{0}=0$. Thus (19) with the induction hypothesis gives $a_{l}=c_{l} z^{b+v}, l=1, \ldots, s$, where $c_{l}$ are constants. We now immediately obtain the equation

$$
\sum_{l=0}^{s} c_{l^{b+v}} u_{s-\imath} u_{s}+z A_{s}^{\prime}=(z+b) A_{s} .
$$

\section{Denote}

$$
A_{s}=A_{s+1}+P_{10} u_{s}+P_{01} u_{s}^{\prime}+P_{20} u_{s}^{2}+P_{11} u_{s} u_{s}^{\prime}+P_{02} u_{s}^{\prime 2},
$$

where $A_{s+1} ; P_{10}, P_{01}$ and $P_{20}, P_{11} P_{02}$ are homogeneous polynomials of degree two; one and zero, respectively, in $u(s-1), u^{\prime}(s-1)$. So $P_{20}, P_{11}, P_{02}$ are polynomials in $z$ satisfying, by $(20)$ and the induction hypothesis,

$$
\begin{aligned}
& z P_{20}^{\prime}-(z+b) P_{20}+\mu P_{11}+c_{0} z^{b+v}=0, \\
& z P_{11}^{\prime}-(b+v) P_{11}+2 z P_{20}+2 \mu P_{02}=0, \\
& z P_{02}^{\prime}+(z-b-2 \nu) P_{02}+z P_{11}=0 .
\end{aligned}
$$

Let $d_{1}, d_{2}$ and $d_{3}$ be the degrees of the polynomials $P_{20}, P_{11}$ and $P_{02}$ respectively. Further, let $c$ be the coefficient of $z^{d_{1}}$ in $P_{20}$. If $d_{1}>b+v-1$, then it follows from (21), that $d_{2}=d_{3}=d_{1}+1$. The second equation of (21) now gives

$$
d_{2} c / \mu-(b+\nu) c / \mu+2 c-2 c=\left(d_{1}-b-\nu+1\right) c / \mu=0,
$$

which is impossible. If $d_{1}<b+v-1$, then $d_{2}=d_{3}=b+v$, and from the second equation of (21) it follows that

$$
-d_{2} c_{0} / \mu+(b+\nu) c_{0} / \mu+2 c_{0}=2 c_{0}=0,
$$

but this, too, is impossible, since $c_{0} \neq 0$. So we are left with the case $d_{1}=b+v-1$. Then we must have $d_{2}=d_{3}=d_{1}+1$. Thus (21) again gives the following contradiction,

$$
d_{2}\left(c-c_{0}\right) / \mu-(b+\nu)\left(c-c_{0}\right) / \mu+2 c+2\left(c_{0}-c\right)=2 c_{0}=0 .
$$

$2^{\circ} \cdot 2 s+1=k$. By substituting $\bar{u}(s)=u(s), \bar{u}_{s+1}=u_{s+1}+\sigma u_{0}$, $\bar{u}_{s+2}=u_{s+2}+\sigma u_{1}, \ldots, \bar{u}_{2 s+2}=u_{2 s+2}+\sigma u_{s+1}$ in (14), we obtain

$$
\begin{aligned}
& \sum_{l=0}^{s} a_{l} \sum_{j=0}^{s-l}\left(u_{j} u_{2 s+2-l-j}^{\prime}-u_{j}^{\prime} u_{2 s+2-l-j}+\sigma\left(u_{j} u_{s+1-l-j}^{\prime}-u_{j}^{\prime} u_{s+1-l-j}\right)\right) \\
+ & A_{s+1}+P_{10}\left(u_{s+1}+\sigma u_{0}\right)+P_{01}\left(u_{s+1}^{\prime}+\sigma u_{0}^{\prime}\right)+P_{20}\left(u_{s+1}+\sigma u_{0}\right)^{2}+ \\
+ & P_{11}\left(u_{s+1}+\sigma u_{0}\right)\left(u_{s+1}^{\prime}+\sigma u_{0}^{\prime}\right)+P_{02}\left(u_{s+1}^{\prime}+\sigma u_{0}^{\prime}\right)^{2}=c(\sigma) z^{b} e^{z},
\end{aligned}
$$

where we have presented $A_{s}$ in the form 
$A_{s}=A_{s+1}+P_{10} u_{s+1}+P_{01} u_{s+1}^{\prime}+P_{20} u_{s+1}^{2}+P_{11} u_{s+1} u_{s+1}^{\prime}+P_{02} u_{s+1}^{\prime 2}$, and here $A_{s+1} ; P_{10}, P_{01}$ and $P_{20}, P_{11}, P_{02}$ are homogeneous polynomials of degree two; one and zero, respectively, in $u(s), u^{\prime}(s)$.

We differentiate this equation with respect to $\sigma$ and put $\sigma=0$. In this way we obtain the equation

$$
\begin{gathered}
\sum_{l=0}^{s} a_{l}\left(u_{0} u_{s+1-l}^{\prime}-u_{0}^{\prime} u_{s+1-l}\right)+P_{10} u_{0}+P_{01} u_{0}^{\prime}+2 u_{0} u_{s+1} P_{20}+ \\
+P_{11}\left(u_{0} u_{s+1}^{\prime}+u_{0}^{\prime} u_{s+1}\right)+2 u_{0}^{\prime} u_{s+1}^{\prime} P_{02}=c^{\prime}(0) z^{b} e^{z} .
\end{gathered}
$$

We see from the induction hypothesis that this equation is impossible. This contradiction completes the proof of step A .

5. Step B. In order to prove the algebraic independence of the functions $u(k+1), u^{\prime}(k+1)$ over $\mathbf{C}(z)$, let us imagine, contrary to this proposition, that these functions are algebraically dependent over $\mathbb{C}(z)$. Then the functions $u(k), \quad u^{\prime}(k), t$ and $v=u_{k+1} / u_{0}$ are also algebraically dependent over $\mathbf{C}(z)$. From step A it follows that $v$ is algebraic over the field $\mathbf{F}=\mathbf{C}\left(z, u(k), u^{\prime}(k), t\right)$. Now

$$
v^{\prime}=\left(u_{0} u_{k+1}^{\prime}-u_{0}^{\prime} u_{k+1}\right) / u_{0}^{2}=t / u_{0}^{2}
$$

is an element of $\mathbf{F}$. Thus Lemma 1 of [2] implies that $v$ itself is also an element of $\mathbf{F}$. Thus there exist two polynomials $Q$ and $P$ in $z, u(k)$, $u^{\prime}(k), t$, having no common factors, such that

$$
Q v-P=0 \text {. }
$$

When we differentiate this equation and multiply the result by $z u_{0}^{2}$, we obtain

$$
z u_{0}^{2} Q^{\prime} v+z Q t-z u_{0}^{2} P^{\prime}=0 .
$$

If $Q^{\prime}=0$, then $Q t=u_{0}^{2} P^{\prime}$, and this is impossible by step A. So it follows that $Q^{\prime} \neq 0$.

The left-hand side of this equation (23) is a polynomial in the same variables as $Q v-P$. That polynomial must be divisible by $Q v-P$, since otherwise we would obtain a contradiction with step $A$ by eliminating $v$ from the equations (22) and (23). This means that there exists a polynomial $R$ in $z, u(k), u^{\prime}(k), t$ such that we have, identically in $z$, $u(k), u^{\prime}(k), t$,

$$
\begin{gathered}
z u_{0}^{2} Q^{\prime}=R Q, \\
z Q t-z u_{0}^{2} P^{\prime}=-R P .
\end{gathered}
$$

If $R$ is not divisible by $u_{0}$, then (24) yields the result that both polynomials $Q$ and $P$ are divisible by $u_{0}^{2}$. This contradiction gives the result $R=u_{0} S$, where $S$ is a polynomial in $z, u(k), u^{\prime}(k), t$. 
Denote

$$
Q=\sum_{i=0}^{m} A_{i} t^{i}, \quad A_{m} \neq 0,
$$

where $A_{i}$ are polynomials in $z, u(k), u^{\prime}(k)$. The first equation (24) gives

$$
u_{0}\left(z A_{m}^{\prime}+m(z-v) A_{m}\right)=S A_{m} .
$$

From this and step A it follows that $S$ cannot contain $t$, and is at most of degree one with respect to $z$ or with respect to $u(k), u^{\prime}(k)$.

First let us assume that $S$ is not divisible by $u_{0}$. Then (24) implies that $Q$ is divisible by $u_{0}$, say $Q=u_{0} Q_{0}$. By (24),

$$
\begin{aligned}
& z u_{0} Q_{0}^{\prime}=\left(S-z u_{0}^{\prime}\right) Q_{0}, \\
& z Q_{0} t-z u_{0} P^{\prime}=-S P .
\end{aligned}
$$

From these equations it follows that $S-z u_{0}^{\prime}$ must be divisible by $u_{0}$, since otherwise $Q_{0}$, and also $P$, would be divisible by $u_{0}$, which is impossible. Thus we obtain

$$
\begin{aligned}
& z Q_{0}^{\prime}=(a z+b) Q_{0}, \\
& z Q_{0} t=z u_{0} P^{\prime}-\left\{(a z+b) u_{0}+z u_{0}^{\prime}\right\} P
\end{aligned}
$$

with constants $a$ and $b$.

Let us denote

$$
Q_{0}=\sum_{i=0}^{m} B_{i} t^{i}, \quad P=\sum_{i=0}^{n} P_{i} t^{i}, \quad B_{m} P_{n} \neq 0,
$$

where $B_{i}$ and $P_{i}$ are polynomials in $z, u(k), u^{\prime}(k)$. Now the first equation of (25) gives

by which

$$
z B_{m}^{\prime}+m(z-v) B_{m}=(a z+b) B_{m},
$$

$$
B_{m}=c_{m} z^{b+m v} e^{(a-m) z} .
$$

The induction hypothesis implies that $a=m$. Further, $b+m v$ must be a non-negative integer, and

$$
B_{m}=c_{m} z^{b+m v}
$$

If $n>m+1$, then the second equation (25) gives, by step $\mathrm{A}$, the following equation

$$
u_{0}\left(z P_{n}^{\prime}+n(z-v) P_{n}\right)-\left\{(m z+b) u_{0}+z u_{0}^{\prime}\right\} P_{n}=0 .
$$

This yields

$$
P_{n}=c_{n} z^{b+n v} u_{0} e^{(m-n) z},
$$


but this contradicts the induction hypothesis. Thus $n=m+1$. By comparing the coefficients of $t^{n}$ in (25), we obtain

$$
c_{m} z^{b+m v+1}=u_{0}\left(z P_{n}^{\prime}+(z-b-n v) P_{n}\right)-z u_{0}^{\prime} P_{n} .
$$

Since $c_{m} \neq 0$ and the right-hand side of this equation is of degree $\geqq 1$ with respect to $u(k), u^{\prime}(k)$, we have obtained a contradiction.

Next let $S=(a z+b) u_{0}$ with constants $a$ and $b$. Then (24) is of the form

$$
\begin{aligned}
& z Q^{\prime}=(a z+b) Q, \\
& z Q t-z u_{0}^{2} P^{\prime}=-(a z+b) u_{0}^{2} P .
\end{aligned}
$$

Thus $Q$ must be divisible by $u_{0}^{2}$. On the other hand, if we denote as before

$$
Q=\sum_{i=0}^{m} A_{i} t^{i}, \quad A_{m} \neq 0,
$$

and use step A, we obtain

Hence,

$$
z A_{m}^{\prime}+m(z-v) A_{m}=(a z+b) A_{m} .
$$

$$
A_{m}=c_{m} z^{b+m v} e^{(a-m) z}, \quad c_{m} \neq 0 .
$$

The induction hypothesis yields $a=m$. Thus $A_{m}$ is a polynomial in z alone, and $Q$ cannot be divisible by $u_{0}^{2}$. This is the desired contradiction, and completes step B.

6. Step C. Here we prove that the functions $u(k+1), u^{\prime}(k+1)$, $e^{\gamma z}, \gamma \in \mathbf{Q}$ and $\gamma \neq 0$, are algebraically independent over $\mathbf{C}(z)$. Let us assume that these functions are algebraically dependent over $\mathbf{C}(z)$.

From Lemma 4 and step B it follows that our assumption implies an equation

$$
P=P_{m} e^{m \gamma z}+P_{0}=0,
$$

where $P_{m}$ and $P_{0}$ are polynomials in $z, u(k+1), u^{\prime}(k+1)$, and one of them is a polynomial in $z$ alone. Further, we have

$$
z P^{\prime}=(a z+b) P, \quad a, b \in \mathbf{Q}
$$

identically in $z, u(k+1), u^{\prime}(k+1), e^{\gamma z}$. So $P_{m}=c_{m} z^{b}$ or $P_{0}=c_{0} z^{b}$, where $c_{m}$ and $c_{0}$ are non-zero constants and $b$ must be a non-negative integer.

We first assume that $\gamma=1$. If $P_{m}=c_{m} z^{b}$, then $a=m$ and $P_{0}$ satisfies the differential equation

$$
z P_{0}^{\prime}=(m z+b) P_{0} .
$$

In the same way as on p. 7 of [7] we can now deduce that 


$$
P_{0}=P_{00}+\sum_{i=1}^{l} Q_{i}\left(u_{0}^{\prime} u_{k+1}-u_{0} u_{k+1}^{\prime}\right)^{i}, \quad Q_{l} \neq 0,
$$

where $P_{00}$ and $Q_{i}$ are polynomials in $z, u(k), u^{\prime}(k)$. Further, as on p. 8 of [7], we obtain

$$
c_{1}^{\prime}(0) z^{b} e^{m z}-\sum_{i=1}^{\ell} i Q_{i}\left(u_{0}^{\prime} y-u_{0} y^{\prime}\right)\left(u_{0}^{\prime} u_{k+1}-u_{0} u_{k+1}^{\prime}\right)^{i-1}=0,
$$

where $y$ is a solution of (4), linearly independent of $u_{0}$. Since $u_{0}^{\prime} y-u_{0} y^{\prime}=c e^{z} / z^{v}, c \neq 0$, we have

$$
c_{1}^{\prime}(0) z^{b+v} e^{(m-1) z}-c \sum_{i=1}^{l} i Q_{i}\left(u_{0}^{\prime} u_{k+1}-u_{0} u_{k+1}^{\prime}\right)^{i-1}=0 .
$$

If $c_{1}^{\prime}(0)=0$, then we have a contradiction with step $B$. If $c_{1}^{\prime}(0) \neq 0$, then $m=1$ and also, by step B, $l=1$. Thus $Q_{1}=d z^{b+v}, d=c_{1}^{\prime}(0) / c$. So it follows that the polynomial

$$
P_{0}=P_{00}+d z^{b+v}\left(u_{0}^{\prime} u_{k+1}-u_{0} u_{k+1}^{\prime}\right)=P_{00}-d z^{b+v} t
$$

satisfies the differential equation

$$
z P_{0}^{\prime}=(z+b) P_{0} .
$$

This is impossible as was proved in step A (see pp. 98-101).

If $P_{0}=c_{0} z^{b}$, then $a=0$ and $P_{m}$ satisfies the differential equation

$$
z P_{m}^{\prime}=(-m z+b) P_{m} .
$$

We can now deduce that $P_{m}$ has the same form (26) as $P_{0}$. The equation analogous to (27) is now of the form

$$
c_{1}^{\prime}(0) z^{b+v}-c e^{(m+1) z} \sum_{i=1}^{l} i Q_{i}\left(u_{0}^{\prime} u_{k+1}-u_{0} u_{k+1}^{\prime}\right)^{i-1}=0 .
$$

The left-hand side of this equation must be divisible by the polynomial $P=P_{m} e^{m z}+c_{0} z^{b}$. This is impossible, and thus the functions $u(k+1)$, $u^{\prime}(k+1), e^{z}$ are algebraically independent over $\mathbf{C}(z)$.

Let $\gamma \neq 0$ be an arbitrary rational number. If $P_{m}=c_{m} z^{b}$, then we can again deduce that $P_{0}$ has the form (26), and the equation analogous to (27) is of the form

$$
c_{1}^{\prime}(0) z^{b+v} e^{m \gamma z}-c e^{z} \sum_{i=1}^{l} i Q_{i}\left(u_{0}^{\prime} u_{k+1}-u_{0} u_{k+1}^{\prime}\right)^{i-1}=0 .
$$

From this it follows that

$$
c_{1}^{\prime}(0) z^{\vee} P_{0} / c_{m}+c e^{z} \sum_{i=1}^{l} i Q_{i}\left(u_{0}^{\prime} u_{k+1}-u_{0} u_{k+1}^{\prime}\right)^{i-1}=0 .
$$


This contradicts with the case $\gamma=1$, which we just proved. If $P_{0}=c_{0} z^{b}$, then we obtain a similar contradiction as before, and thus step C holds. Our Lemma 1 is now proved.

7. We now denote $u_{0, j}(z, \nu, \mu)=u_{0, j}, \quad u_{j, 0}(z, \nu, \mu)=u_{j, 0}$, $j=0,1, \ldots$ In the following let the notations $u(m, n)$ and $u^{\prime}(m, n)$ denote the functions $u_{i, 0}, \quad u_{0, j}$ and $u_{i, 0}^{\prime}, \quad u_{0, j}^{\prime}, \quad i=0,1, \ldots, m$, $j=1,2, \ldots, n$, respectively.

From $(5)$ and $(1)$ it follows that the functions $u(p, n)$ satisfy the following system of differential equations

$$
\begin{gathered}
u_{0, j}^{\prime \prime}+(\nu / z-1) u_{0, j}^{\prime}-(\mu / z) u_{0, j}-(1 / z) u_{0, j-1}=0, \quad u_{0,-1} \equiv 0, \\
\text { (29) } u_{i, 0}^{\prime \prime}+(\nu / z-1) u_{i, 0}^{\prime}-(\mu / z) u_{i, 0}+(1 / z) u_{i-1,0}^{\prime}=0, \quad u_{-1,0} \equiv 0, \\
i=0,1, \ldots, p, \quad j=1,2, \ldots, n .
\end{gathered}
$$

Lemmas 2 and 3 will now be proved simultaneously. The proofs will be performed by induction. If $m=0$, then Lemmas 2 and 3 follow from Lemma 1, which we just proved.

Now, let us assume that the functions

$$
u(m, n), u^{\prime}(m, n), e^{\gamma z}
$$

are algebraically independent over $\mathbf{C}(z)$. Using this assumption, we prove that the functions

$$
u(m+1, n), u^{\prime}(m+1, n), e^{\gamma z},
$$

too, are algebraically independent over $\mathbf{C}(z)$. Let us assume the contrary case that the functions (31) are algebraically dependent over $\mathbf{C}(z)$. We shall prove that this leads to a contradiction.

The proof is divided into three steps, called here A 1, B 1 and C 1 .

8. Step A 1. By analogy with step A we denote

$$
t=u_{00} u_{m+1,0}^{\prime}-u_{00}^{\prime} u_{m+1,0},
$$

and prove that the functions

$$
u(m, n), \quad u^{\prime}(m, n)
$$

and $t$ are algebraically independent over $\mathbf{C}(z)$. Otherwise we would have an equation

$$
P=\sum_{i=0}^{l} P_{i} t^{i}=0
$$

where $P$ is an irreducible polynomial in $z, t$ and $(32)$; and $P_{i}$, $i=0,1, \ldots, l$, are polynomials in $z$ and $(32)$ such that $P_{l} \neq 0$. By the induction hypothesis, $l \geqq 1$. 
In a completely analogous way to step A (pp. 97-98) we can now deduce that $P$ is of the form

$$
P=c_{0} z^{b+v} t+P_{0}=0
$$

and satisfies

$$
z P^{\prime}=(z+b) P, \quad b \in \mathbf{Q},
$$

identically in $z, t$ and (32). Further, $P_{0}$ is a homogeneous polynomial of degree two with respect to $(32)$.

Since (34) is an identity, it follows that (34) holds if we replace the solution $u(m+1, n)$ by any other solution $\bar{u}(m+1, n)$ of $(29)$ with $p=$ $m+1$.

We now write $s=[\mathrm{m} / 2]$. By analogy with reasoning employed on pp. 98-99 we can deduce that $P$ must be of the form

$$
\begin{gathered}
P=\sum_{l=0}^{s} a_{l} \sum_{j=0}^{s-l}\left(u_{j 0} u_{m+1-l-j, 0}^{\prime}-u_{j 0}^{\prime} u_{m+1-l-j, 0}\right)+A ; \\
a_{0}=c_{0} z^{b+v} \neq 0,
\end{gathered}
$$

where $A$ is a homogeneous polynomial of degree two with respect to $u(0, n), u^{\prime}(0, n), u_{j 0}, u_{j 0}^{\prime}, j=1,2, \ldots, m-s$. From (34) it now follows that

$$
\begin{gathered}
\sum_{l=1}^{s}\left(z a_{l}^{\prime}-(b+v) a_{l}-a_{l-1}\right) P_{l}-\sum_{l=0}^{s} a_{l} u_{s-l, 0} u_{m-s, 0}^{\prime}+z A^{\prime}= \\
(z+b) A,
\end{gathered}
$$

where we have denoted

$$
P_{l}=\sum_{j=0}^{8-l}\left(u_{j, 0} u_{m+1-l-j, 0}^{\prime}-u_{j, 0}^{\prime} u_{m+1-l-j, 0}\right) .
$$

If $s>0$, which means $m>1$, then the induction hypothesis yields

$$
z a_{1}^{\prime}-(b+\nu) a_{1}-c_{0} z^{b+v}=0 .
$$

If $b+v=0$, then $z a_{1}^{\prime}=c_{0}$. But this is impossible. If $b+v>0$, then $a_{1}=d z^{i}+e z^{i+1}+\ldots, \quad d \neq 0, \quad 1 \leqq i \leqq b+v$. Thus we obtain

$$
i d-(b+\nu) d-\delta_{i, b+v} c_{0}=0,
$$

and this leads to a contradiction.

If $m=1$, then $P$ is of the form

$P=c_{0} z^{b+v} t+A_{1}+P_{10} u_{10}+P_{01} u_{10}^{\prime}+P_{20} u_{10}^{2}+P_{11} u_{10} u_{10}^{\prime}+P_{02} u_{10}^{\prime 2}$, where $A_{1} ; P_{10}, P_{01}$ and $P_{20}, P_{11}, P_{02}$ are homogeneous polynomials of degree two; one and zero with respect to $u(0, n), u^{\prime}(0, n)$. When in (34) we replace $u(2, n)$ by the solution $\bar{u}(0, n)=u(0, n), \bar{u}_{i 0}=u_{i 0}+\sigma u_{i-1,0}$, 
$i=1,2$, integrate (34), differentiate the result with respect to $\sigma$ and put $\sigma=0$, we obtain

$$
\begin{gathered}
c_{0} z^{b+v}\left(u_{00} u_{10}^{\prime}-u_{00}^{\prime} u_{10}\right)+P_{10} u_{00}+P_{01} u_{00}^{\prime}+2 P_{20} u_{10} u_{00} \\
+P_{11}\left(u_{10} u_{00}^{\prime}+u_{10}^{\prime} u_{00}\right)+2 P_{02} u_{10}^{\prime} u_{00}^{\prime}=c^{\prime}(0) z^{b} e^{z} .
\end{gathered}
$$

The induction hypothesis tells us that this is impossible.

Next, let $m=0$, and let $r=[n / 2]$. If $r>0$, then we obtain, using the induction hypothesis and a similar technique as on pp. 98-99,

$$
P=c_{0} z^{b+v} t+\sum_{l=0}^{r-1} a_{l} \sum_{j=0}^{r-1-l}\left(u_{0 j} u_{0, n-l-j}^{\prime}-u_{0 j}^{\prime} u_{0, n-l-j}^{\prime}\right)+A_{r},
$$

where $a_{l}=a_{l}(z)$ are polynomials in $z$ and $A_{r}$ is a homogeneous polynomial of degree two with respect to $u(0, n-r), u^{\prime}(0, n-r)$. Now either $2 r=n$ or $2 r=n-1$.

$1^{\circ} . \quad 2 r=n$. The substitution $\quad \bar{u}(0, r-1)=u(0, r-1), \bar{u}_{0 j}=$ $u_{0 j}+\sigma u_{0, j-r}, j=r, \ldots, n$, in (34) now gives, after similar steps as before,

$$
\begin{gathered}
\sum_{l=0}^{r-1} a_{l}\left(u_{00} u_{0, r-l}^{\prime}-u_{00}^{\prime} u_{0, r-l}\right)+P_{10} u_{00}+P_{01} u_{00}^{\prime}+2 P_{20} u_{0 r} u_{00}+ \\
+P_{11}\left(u_{0 r} u_{00}^{\prime}+u_{0 r}^{\prime} u_{00}\right)+2 P_{02} u_{0 r}^{\prime} u_{00}^{\prime}=c^{\prime}(0) z^{b} e^{z},
\end{gathered}
$$

where we have presented $A_{r}$ in the typical form

$$
A_{r}=A_{r+1}+P_{10} u_{0 r}+P_{01} u_{0 r}^{\prime}+P_{20} u_{0 r}^{2}+P_{11} u_{0 r} u_{0 r}^{\prime}+P_{02} u_{0 r}^{\prime 2} .
$$

From the induction hypothesis it follows that $a_{0}(z) \equiv 0$.

$2^{\circ} .2 r=n-1$. By the substitution $\bar{u}(0, r)=u(0, r), \quad \bar{u}_{0 j}=$ $u_{0 j}+\sigma u_{0, j-r-1}, j=r+1, \ldots, n$, in (34) we get

$$
P=c_{0} z^{b+v} t+\sum_{l=0}^{r} a_{l} \sum_{j=0}^{r-l}\left(u_{0 j} u_{0,2 r+1-l-j}^{\prime}-u_{0 j}^{\prime} u_{0,2 r+1-l-j}\right)+A
$$

where $A$ is a homogeneous polynomial of degree two with respect to $u(0, r), u^{\prime}(0, r)$. By $(34)$ we have

$$
\begin{gathered}
-c_{0} z^{b+v} u_{00} u_{00}^{\prime}+\sum_{l=0}^{r}\left(z a_{l}^{\prime}-(b+v) a_{l}\right) P_{l}+\sum_{l=0}^{r} a_{l} u_{0, r-l} u_{0 r}+z A^{\prime}= \\
(z+b) A,
\end{gathered}
$$

where

$$
P_{l}=\sum_{j=0}^{r-l}\left(u_{0 j} u_{0,2 r+1-l-j}^{\prime}-u_{0 j}^{\prime} u_{0,2 r+1-l-j}\right) .
$$

By the induction hypothesis we get $a_{l}=d_{l} z^{b+v}$, where $d_{l}$ are constants. As on pp. 100-101 we obtain a contradiction if $d_{0} \neq 0$. Thus $a_{0}(z) \equiv 0$. Thus we see that the functions $u_{0, n}, u_{0, n}^{\prime}$ do not occur in $P$. 
By continuing in the same way we can deduce that none of the functions $u_{0, i}, u_{0, i}^{\prime},[i / 2]>0$, can occur in $P$. Thus we finally see that $P$ must be of the form

$$
P=c_{0} z^{b+v} t+c z^{b+v}\left(u_{00} u_{01}^{\prime}-u_{00}^{\prime} u_{01}\right)+P_{20} u_{00}^{2}+P_{11} u_{00} u_{00}^{\prime}+P_{02} u_{00}^{\prime 2},
$$

where $P_{20}, P_{11}, P_{02}$ are polynomials in $z$. These polynomials must satisfy the equations (see p. 100)

$$
\begin{aligned}
& z P_{20}^{\prime}-(z+b) P_{20}+\mu P_{11}+c z^{b+v}=0, \\
& z P_{11}^{\prime}-(b+v) P_{11}+2 z P_{20}+2 \mu P_{02}-c_{0} z^{b+v}=0, \\
& \approx P_{02}^{\prime}+(z-b-2 \nu) P_{02}+z P_{11}=0 .
\end{aligned}
$$

Let us assume, at first, that $c=0$. If $d_{1}, d_{2}$ and $d_{3}$ are the degrees of $P_{20}, P_{11}$ and $P_{02}$ respectively, then (41) gives $d_{2}=d_{3}=d_{1}+1=$ $b+v$. If $d$ is the coefficient of $z^{d_{3}}$ in $P_{02}$, then it follows from (41) that

$$
-d_{2} d+(b+\nu) d-2 \mu d+2 \mu d-c_{0}=-c_{0}=0,
$$

which is impossible, since $c_{0} \neq 0$. This gives step A 1 of Lemma 2 (we have not yet used the assumption $\nu \neq 1,2, \ldots$ of Lemma 3 ).

Next, let $c \neq 0$. The degrees $d_{2}=d_{3}$ and $d_{1}+1$ cannot exceed $b+v$. Further, since $v \neq 1,2, \ldots$ in Lemma 3, it follows from (41) that $P_{11}$ and $P_{02}$ are not $\equiv 0$. Now let $P_{02}=k z^{i}+l z^{i+1}+\ldots, k \neq 0$. By putting $P_{11}=k_{1} z^{i-1}+l_{1} z^{i}+\ldots$ and by using the last equation (41) we obtain

$$
i k-(b+2 v) k+k_{1}=0 .
$$

This yields $k_{1} \neq 0$, since $i-b-2 v=i-n_{0}-v \neq 0, \quad n_{0}=b+v$. From the second equation (4l) it follows that $P_{20}=k_{2} z^{i-2}+l_{2} z^{i-1}+\ldots$, where $k_{2} \neq 0$. Then we use the first equation (41), which gives

$$
(i-2) k_{2}-b k_{2}=0 \text {. }
$$

Thus $b$ is an integer, and so $v=n_{0}-b$ must also be an integer. This contradiction completes the proof of step A 1 .

9. Step B 1. Here we can use a word to word repetition of step B (pp. 101-103), now defining $v=u_{m+1,0} / u_{00}$. We thus obtain the algebraic independence of the functions (32) and $u_{m+1,0}, u_{m+1,0}^{\prime}$ over $\mathbf{C}(z)$.

10. Step C 1. The functions $(30)$ and $u_{m+1,0}, u_{m+1,0}^{\prime}$ can now be proved to be algebraically independent over $\mathbf{C}(z)$ in a completely analogous way to the proof of step $C$. (It should be noted that Lemma 4 remains valid if we replace the functions $u(n), u^{\prime}(n)$ by the functions $u(m+1, n)$, $u^{\prime}(m+1, n)$.) Thus Lemmas 2 and 3 are true. 
11. The functions $u(m, n)$ and $u^{\prime}(m, n)$ are $E$-functions (for the definition of $E$-functions see p. 33 of [6]). Further, by (29), the functions $u(0, n), u^{\prime}(0, n), e^{z} ; u(m, 0), u^{\prime}(m, 0), e^{z}$ and $u(m, n), u^{\prime}(m, n)$, $e^{z}$ satisfy

$$
\begin{array}{rlrl}
\left(u_{0 j}\right)^{\prime}=u_{0 j}^{\prime}, \quad\left(u_{0 j}^{\prime}\right)^{\prime} & =(1-\nu / z) u_{0 j}^{\prime}+(\mu / z) u_{0 j}+(1 / z) u_{0, j-1}, & y^{\prime}=y, \\
& u_{0,-1} \equiv 0, \quad j=0,1, \ldots, n ; \\
\left(u_{i 0}\right)^{\prime}=u_{i 0}^{\prime}, \quad\left(u_{i 0}^{\prime}\right)^{\prime}=(1-\nu / z) u_{i 0}^{\prime}+(\mu / z) u_{i 0}-(1 / z) u_{i-1,0}^{\prime}, & y^{\prime}=y, \\
& u_{-1,0} \equiv 0, \quad i=0,1, \ldots, m, &
\end{array}
$$

and

$\left(u_{0 j}\right)^{\prime}=u_{0 j}^{\prime}, \quad\left(u_{0 j}^{\prime}\right)^{\prime}=(1-\nu / z) u_{0 j}^{\prime}+(\mu / z) u_{0 j}+(1 / z) u_{0, j-1}, \quad u_{0,-1} \equiv 0$, $\left(u_{i 0}\right)^{\prime}=u_{i 0}^{\prime}, \quad\left(u_{i 0}^{\prime}\right)^{\prime}=(1-\nu / z) u_{i 0}^{\prime}+(\mu / z) u_{i 0}-(1 / z) u_{i-1,0}^{\prime}, \quad u_{-1,0} \equiv 0$, $y^{\prime}=y ; \quad i=0,1, \ldots, m, \quad j=1,2, \ldots, n$.

Thus we can end our paper by establishing that the truth of our theorems follows from our lemmas and Sidlovskiî's theorem [5].

\section{References}

[1] Belogrivov, I. I.: On transcendence and algebraic independence of the values of Kummer's functions. - Siberian Math. J. 12, 1971, 690-705.

[2] Mahler, K.: Applications of a theorem by A. B. Shidlovski. - Proc. Roy. Soc. Ser. A $305,1968,149-173$.

[3] Oleinikov, V.A.: On the transcendence and algebraic independence of the values of certain $E$-functions. - Vestn. Mosk. Gos. Un-ta, Ser. I, No. 6, 1962, 34-38.

[4] ŠIdlovskiř, A. B.: On criteria of algebraic independence of values of a class of integral functions. - Amer. Math. Soc. Transl. (2) 22, 1962, 339 - 370.

[5] -" - On the transcendence and algebraic independence of the values of $E$ functions related by an arbitrary number of algebraic equations over the field of rational functions. - Amer. Math. Soc. Transl. (2) 50, 1966, $141-$ 177.

[6] Siegel, C. L.: Transcendental numbers. - Princeton University Press, Princeton, 1949.

[7] VäÄNÄNEN, K.: On the transcendence and algebraic independence of the values of certain $E$-functions. - Ann. Acad. Sci. Fenn. Ser. A I 537, 1973, 1-15.

University of Oulu

Department of Mathematics

SF-90100 Oulu 10

Finland

Received 1 October 1974 